\title{
Computed tomography imaging study of the superior attachment of the uncinate process*
}

\author{
Bruno Netto', Otávio Bejzman Piltcher², Camila Degen Meotti³, \\ Jaqueline Lemieszek4, Gustavo Rassier Isolan ${ }^{5}$ \\ ' Graduate Program in Medicine: Surgical Sciences, Universidade Federal do Rio Grande do Sul (UFRGS), Porto Alegre, RS, Brazil \\ 2 School of Medicine, UFRGS, Porto Alegre, RS, Brazil \\ ${ }^{3}$ Department of Otorhinolaryngology, Hospital de Clínicas de Porto Alegre (HCPA), Porto Alegre, RS, Brazil \\ ${ }^{4}$ Department of Radiology, HCPA, Porto Alegre, RS, Brazil \\ ${ }^{5}$ Department of Neurosurgery, HCPA, Porto Alegre, RS, Brazil
}

Rhinology 53: 187-191, 2015

DOl:10.4193/Rhino 14.174

*Received for publication:

July 14,2014

Accepted: January 8, 2015

\begin{abstract}
Background: The superior attachment of the uncinate process (SAUP) is an important landmark in the nasofrontal recess, but its anatomical distribution is still unclear. The objective of this study was to assess the location and number of superior attachments of the uncinate process on computed tomography (CT) images.
\end{abstract}

Methodology: A cross-sectional, retrospective study conducted at the Department of Radiology, Hospital de Clínicas de Porto Alegre, Brazil. Sinus CT scans of adult patients with no history of sinonasal surgery, craniofacial fractures, malignant neoplasms, or malformations were examined for identification of the site of SAUP.

Results: 152 CT scans (304 sides) were assessed. The SAUP was appropriately identified in 292 sides (96\%). Six distinct SAUP patterns were identified: to the lamina papyracea or anterior ethmoidal cells $(193,63.48 \%)$; to the middle turbinate $(19,6.25 \%)$; to the skull base (19, 6.25\%); to the lamina papyracea and middle turbinate $(29,9.53 \%)$; to the lamina papyracea and skull base (29, $9.53 \%)$; and to the skull base and middle turbinate $(3,0.98 \%)$.

Conclusion: The site of superior attachment of the uncinate process is highly variable, but is in contact with the lateral nasal wall in $82.5 \%$ of the cases.

Key words: nasal cavity, paranasal sinuses, ethmoid bone

\section{Introduction}

The uncinate process (UP) of the ethmoid bone is a thin, hook-shaped bony projection of the lateral nasal wall located between the middle and inferior turbinates, medial to the ostium of the maxillary sinus ${ }^{(1-4)}$. Its inferior aspect attaches to the ethmoidal process of the inferior turbinate. Its middle portion is adjacent to the lacrimal bone anteriorly and, posteriorly, delimits the space or gap known as the semilunar hiatus. Lateral to the middle portion of the uncinate process lies the infundibulum, which contains the true ostium of the maxillary sinus. The superior portion of the uncinate process is anatomically variable, and its pattern of distribution has yet to be fully established. The uncinate process is an essential anatomical landmark for endoscopic paranasal sinus surgery. Its resection - uncinectomy - is paramount to obtaining access to the maxillary and frontal sinuses and, if performed incompletely, may lead to complications and even revision surgery ${ }^{(5)}$. With recent advancements in transnasal endoscopic neurosurgery, uncinectomy has also become a part of the transmaxillary corridor approach, which enables endoscopic access to the infratemporal and pterygopalatine fossae ${ }^{(6,7)}$. The uncinate also plays an important role in conforming the nasofrontal recess ${ }^{(8)}$, i.e., the three-dimensional 
space that connects the frontal sinus and nasal cavity. The superior attachment of the uncinate process (SAUP), located within the nasofrontal recess, is a key structure to the frontal sinus drainage pathway and plays a relevant role in surgical approaches to the frontal sinus ${ }^{(9)}$. Landsberg and Friedmann ${ }^{(10)}$ describe the SAUP as the most important landmark in frontal recess surgery. Although a series of studies conducted in recent years have sought to elucidate the pattern of superior attachment of the uncinate, they have yielded conflicting results and provided insufficient evidence to consolidate knowledge on the matter. Furthermore, no computed tomography (CT)-based studies of the SAUP have been conducted in Brazil.

In recent years, the potential role of the uncinate process in the pathophysiology of sinusitis has also been the subject of increasing research interest. As it is located at the site of frontal, maxillary, and anterior ethmoid sinus drainage, the pattern of SAUP may interfere with the normal physiology of these sinuses and contribute to the development of sinus disease. However, no studies have sought to assess whether different SAUP types are associated with the presence of sinus conditions.

\section{Materials and methods}

This was a cross-sectional, retrospective study conducted at the Department of Radiology, Hospital de Clínicas de Porto Alegre (HCPA), on paranasal sinus CT scans obtained between March 2010 and December 2012 and available through the HCPA picture archiving and communication system. Patients younger than 18 years and those with a history of sinonasal surgery, craniofacial malformations, fractures, or malignant neoplasm were excluded from the sample. Based on the standard error of SAUP proportion estimation reported in Landsberg et al. ${ }^{(10)}$, the sample size required for an error rate of $5 \%$ is 300 sides. This study was approved by the HCPA Research Ethics Committee and the investigators signed a Data Use Agreement.

\section{Imaging acquisition and analysis}

CT scans of the paranasal sinuses were obtained with multislice (multi-detector array) scanners (Philips Brilliance 16 Power, software version 2.3.0, the Netherlands; and GE Healthcare BrightSpeed S 10BW, software version 27.7, United States) and the IMPAX 6.3 picture archiving and communications system and processed in a Volume Viewing 3.1 software workstation for analysis of 1-mm slices. CT imaging was performed using a specific protocol (Phillips 120 kV, 150 mA; GE 120 kV, 250 mA). All scans were examined simultaneously by the same radiologist and otorhinolaryngologist. The SAUP was identified on coronal slices by focusing on the nasofrontal recess area, scrolling the image anteriorly and posteriorly to identify the superior aspect of the bony lamina of the uncinate process, and following it to its superior attachment. The drainage site of the frontal sinuses

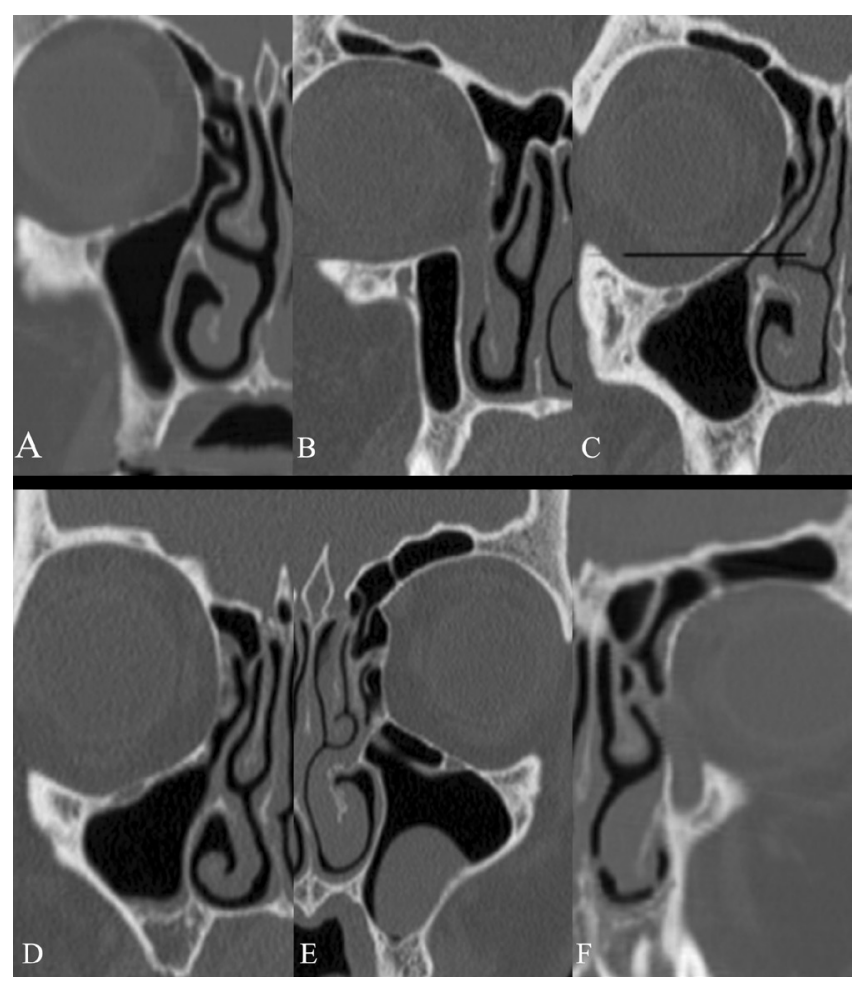

Figure 1. Patterns of superior attachment of the uncinate process. A) To the lamina papyracea; $B$ ) to the middle turbinate; C) to the skull base; D) to the lamina papyracea and middle turbinate; E) to the lamina papyracea and skull base; and F) to the skull base and middle turbinate.

was identified and Lund-Mackay scores calculated. Each side was examined individually.

\section{Statistical Analysis}

All statistical analyses were carried out in the Statistical Package for the Social Sciences (SPSS) 20.0 software environment. Demographic variables were expressed as means, standard deviations, and ranges (numerical variables) or frequencies and percentages (categorical variables).

\section{Results}

A review of records was conducted of 187 patients for whom paranasal sinus $\mathrm{CT}$ scans were available through the HCPA picture archiving and communication system. Of these 187 patients, 35 did not meet the inclusion criteria ( 28 due to a history of craniofacial surgery, five with a history of craniofacial fractures, one with cleft palate, and one with a sinonasal neoplasm). Therefore, the sinus CT scans of 152 patients (304 sides) were assessed. Over half of the included patients were women (57.8\%), and mean age was 46.6 years (range, 19-88 years).

The SAUP was successfully identified in 292 sides (96.1\%). In the 12 remaining sides (3.9\%), mucosal thickening or build-up of secretion within the ethmoid cells precluded clear identification of the SAUP. Six distinct SAUP patterns were identified (Table 
Table 1. Sample profile and summary of findings.

\begin{tabular}{|c|c|}
\hline Feature & Number (\%) \\
\hline Sex, M/F & $\begin{array}{c}129(42.2 \%) / \\
175(57.8 \%)\end{array}$ \\
\hline Age in years, mean (range) & $46.6(19-88)$ \\
\hline Single SAUP, N (\%) & $199(65.5 \%)$ \\
\hline Identical bilateral SAUP, N (\%) & $91(59.9 \%)$ \\
\hline \multicolumn{2}{|l|}{ Type of attachment, number of sides (\%) } \\
\hline To lamina papyracea/anterior ethmoidal cells & $193(63.5 \%)$ \\
\hline To lamina papyracea and skull base & $29(9.5 \%)$ \\
\hline To lamina papyracea and middle turbinate & $29(9.5 \%)$ \\
\hline To skull base & $19(6.3 \%)$ \\
\hline To middle turbinate & $19(6.3 \%)$ \\
\hline To skull base and middle turbinate & $3(0.9 \%)$ \\
\hline \multicolumn{2}{|l|}{ Frontal sinus drainage, number of sides (\%) } \\
\hline Middle meatus & $271(89.9 \%)$ \\
\hline Maxillary infundibulum & $33(10.8 \%)$ \\
\hline
\end{tabular}

1, Figure 1). In most cases, the UP had a single superior attachment (199 sides, 65.46\%), which was identical on both sides (91 patients, $59.86 \%)$. In $82,5 \%$ of cases, SAUP was attached directly or indirectly to the lamina papyracea.

The site of frontal sinus drainage was identified in 299 sides
(98.3\%), and was most commonly to the middle meatus (271 sides, $89.1 \%)$. When the frontal sinus drained into the maxillary sinus (33 sides, 10.8\%), the SAUP was most commonly to the middle turbinate ( 15 sides, $45.4 \%$ ), skull base ( 8 sides, $24.2 \%$ ), and to the lamina papyracea and skull base (6 sides, $18.25 \%$ ).

\section{Discussion}

Since 2000, six studies (Table 2) have sought to assess the sites of superior attachment of the uncinate process; all found broad variability in attachment patterns ${ }^{(10-15)}$. Despite different findings, all six studies were unanimous as to the lamina papyracea being the most common site of SAUP. There was substantial variability in all other attachment sites, which prevented us from drawing definitive conclusions as to whether an actual pattern of attachment exists and repeats across different populations. In the present study, structures of the lateral nasal wall also accounted for the majority of superior UP attachment sites. We chose to include attachment to the lamina papyracea directly and to the anterior ethmoidal cells, including agger nasi cells, in the same group. This classification strategy has been used by other authors previously ${ }^{(12,14,15)}$ and makes interpretation of CT findings and data analysis easier. Nevertheless, in our sample, $73 \%$ of cases with a type 1 attachment (to the lamina papyracea/anterior ethmoidal cells) did not exhibit direct attachment to the lamina papyracea, but rather attachment to an adjacent ethmoidal cell. This finding is relevant to surgical approaches to

Table 2. Prior SAUP studies and the attachment patterns and prevalence rates described therein.

\begin{tabular}{|c|c|c|c|c|c|c|c|c|c|}
\hline \multirow{2}{*}{ Author } & \multirow{2}{*}{ Country } & \multirow{2}{*}{ N (sides) } & \multicolumn{7}{|c|}{ Site of superior attachment of the uncinate process (\%) } \\
\hline & & & LP & MT & SB & AN & LP+MT & LP+SB & MT+SB \\
\hline Landsberg ${ }^{(10)}$ & Israel & 288 & 52 & 1.4 & 10.6 & 18.5 & 17.5 & & \\
\hline Yang ${ }^{(11) *}$ & China & 160 & 41 & 19 & 16 & 11 & & & \\
\hline Ercan ${ }^{(12)}$ & Turkey & 361 & 62.6 & 19.9 & 14.4 & & 3.1 & & \\
\hline Zhang ${ }^{(13) * *}$ & China & 42 & 33 & & 4 & & 21 & 31 & 2 \\
\hline $\operatorname{Han}^{(14)}$ & China & 404 & 53 & 5.2 & 2.2 & & 8.7 & 27.5 & 3.5 \\
\hline Liu ${ }^{(15)}$ & Taiwan & 264 & 70.4 & 10.2 & 6.1 & & 4.9 & 7.6 & 0.8 \\
\hline Netto (this study) & Brazil & 304 & 63.5 & 6.3 & 6.3 & & 9.5 & 9.5 & 0.9 \\
\hline
\end{tabular}

LP, lamina papyracea; MT, middle turbinate; SB, skull base; AN, agger nasi. * Not included, "bifurcating superior attachment" group, accounting for $13 \%$ of sides. ** Not included, one case (2\%) of triple attachment (LP+MT+SB) 
the uncinate process, which can be handled much more safely when it is not in direct contact with the lamina papyracea. No similar finding was reported in previous studies, perhaps due to different author criteria or to the lack of a standardized scheme for reporting of attachment sites.

Exact determination of attachment sites is feasible in most cases, but can be challenging due to reduced thickness of the bony lamina of the uncinate process and to the complexity of the cell system of the ethmoid labyrinth, where even slight mucosal thickening or fluid build-up may mimic a non-existent or misplaced attachment. Determination of the site of SAUP is most challenging in patients with pan-sinus disease or extensive nasal polyposis, as reliable examination of the superior aspect of the uncinate may be infeasible in this setting, even though these cases are those in which an accurate understanding of patient anatomy is most important to the surgeon.

Regarding the variability of results among prior studies, ethnicity may have been a determining factor, as the frequencies reported in some Chinese studies were different from those of studies conducted elsewhere. However, the lack of a standardised scheme for description of attachment sites limits comparison across studies, as authors have chosen to report attachment patterns according to their own classifications. The results of the present study were similar to those reported by Liu in a Taiwanese investigation ${ }^{(15)}$. This may have been due to the similarity between the classification scheme used herein and that of the Liu study, enabling easy comparison.

There is a need for development of a standardized SAUP classification that can be used in future studies. In our opinion, this new classification must be straightforward and focus on the key structures of the nasofrontal recess. Classification schemes proposed thus far have used excessively complex descriptions for attachment sites (e.g. "junction of middle turbinate with cribriform plate" and "posteromedial wall of the agger nasi cell") and failed to include well-established patterns, such as double attachment to the lamina papyracea and middle turbinate or middle turbinate and skull base. Our research shows that any attachment pattern can be described in relation to the key structures of the nasofrontal recess: lamina papyracea, skull base, and middle turbinate. Therefore, we believe the classifi- cation proposed herein is both more appropriate for research purposes (from an educational perspective) and easier to use in clinical settings.

There is broad consensus among studies as to the lamina papyracea and anterior ethmoidal cells being the most common sites of SAUP. However, there are major discrepancies as to other attachment patterns and no consensus on their classification. In the present study, the SAUP was most commonly to the lateral structures of the nasal cavity, in direct or indirect contact with the lamina papyracea.

\section{Conclusion}

We propose that the classification scheme employed in this study be used as a reference framework for future SAUP studies. A standardized classification of SAUP patterns is essential for comparison of results among studies and to enable more consistent progress in our anatomical understanding of the SAUP and its clinical repercussions.

Although previous studies point to the lamina papyracea as the most frequent site of SAUP, we note that it attaches chiefly to anterior ethmoidal cells, with only indirect contact with the lamina papyracea. As for the other types of SAUP, there is great discrepancy and no consensus on classification. The middle meatus is the most prevalent site of drainage of the frontal sinus, as reported in previous studies.

\section{Acknowledgements}

We thank the departments of otorhinolaryngology and radiology, Hospital de Clínicas de Porto Alegre, for making this study possible.

\section{Author contributions}

BN: study design and acquisition, analysis, and interpretation of data; OBP: study design, manuscript review and final approval; CDM: study design and acquisition, analysis, and interpretation of data; JL: acquisition, analysis, and interpretation of data; GRI: study design, manuscript review and final approval.

\section{Conflict of interest}

The authors declare there are no conflicts of interest.

\section{References}

1. Bailey BJ, Johnson JT, Newlands SD. Head \& Neck Surgery: Otolaryngology. Philadelphia PA: Lippincott Williams \& Wilkins, 2006; 308.

2. Snow JB, Wackym PA, Ballenger JJ. Ballenger's Manual of Otorhinolaryngology Head and Neck Surgery. Shelton, CT: PMPHUSA, 2009; 461-463.

3. Flint PW, Haughey BH, Lund VJ, et al. Cummings Otolaryngology - Head and Neck Surgery: Head and Neck Surgery,
3-Volume Set. Philadelphia, PA: Elsevier Health Sciences, 2010; 3672.

4. Berger G, Eviatar E, Kogan T, Landsberg R. The normal uncinate process: histology and clinical relevance. Eur Arch Otorhinolaryngol. 2013; 270: 959-964.

5. Richtsmeier WJ. Top 10 reasons for endoscopic maxillary sinus surgery failure. Laryngoscope. 2001; 111: 1952-1956.

6. Schwartz TH, Fraser JF, Brown S, Tabaee A Kacker A, Anand VK. Endoscopic cranial base surgery: classification of operative approaches. Neurosurgery. 2008; 62: 991 1002; discussion 1002-1005

7. Isolan GR, de Aguiar PH, Laws ER, Strapasson AC, Piltcher O. The implications of microsurgical anatomy for surgical approaches to the sellar region. Pituitary. 2009; 12: 360-367.

8. Friedman M, Landsberg $R$, Schults RA, Tanyeri H, Caldarelli DD. Frontal sinus surgery: endoscopic technique and prelimi- 
nary results. Am J Rhinol. 2000; 14: 393-403.

9. Turgut S, Ercan I, Sayin I, Basak M. The relationship between frontal sinusitis and localization of the frontal sinus outflow tract: a computer-assisted anatomical and clinical study. Arch Otolaryngol Head Neck Surg. 2005; 131: 518-522.

10. Landsberg R, Friedman M. A computerassisted anatomical study of the nasofrontal region. Laryngoscope. 2001; 111: 21252130

11. Yang QT, Shi JB, Kang Z, et al. [Computerassisted anatomical study of nasofrontal region]. Zhonghua Er Bi Yan Hou Ke Za Zhi. 2004; 39: 349-352.

12. Ercan I, Cakir BO, Sayin I, Basak M, Turgut S. Relationship between the superior attachment type of uncinate process and pres- ence of agger nasi cell: a computer-assisted anatomic study. Otolaryngol Head Neck Surg. 2006; 134: 1010-1014.

13. Zhang L, Han D, Ge W, et al. Anatomical and computed tomographic analysis of the interaction between the uncinate process and the agger nasi cell. Acta Otolaryngol 2006; 126: 845-852.

14. Han D, Zhang L, Ge W, Tao J, Xian J, Zhou B. Multiplanar computed tomographic analysis of the frontal recess region in Chinese subjects without frontal sinus disease symptoms. ORL J Otorhinolaryngol Relat Spec. 2008; 70: 104-112.

15. Liu SC, Wang CH, Wang HW. Prevalence of the uncinate process, agger nasi cell and their relationship in a Taiwanese population. Rhinology. 2010; 48: 239-244.

\section{Bruno Netto}

Universidade Federal do Rio Grande do Sul

Rua Tiradentes, 855/308,

Centro 96508-772

Cachoeira do Sul, RS

Brazil

Tel: +55-51-3722 4106

E-mail: brunonetto@gmail.com

\section{ADVERTISEMENT}

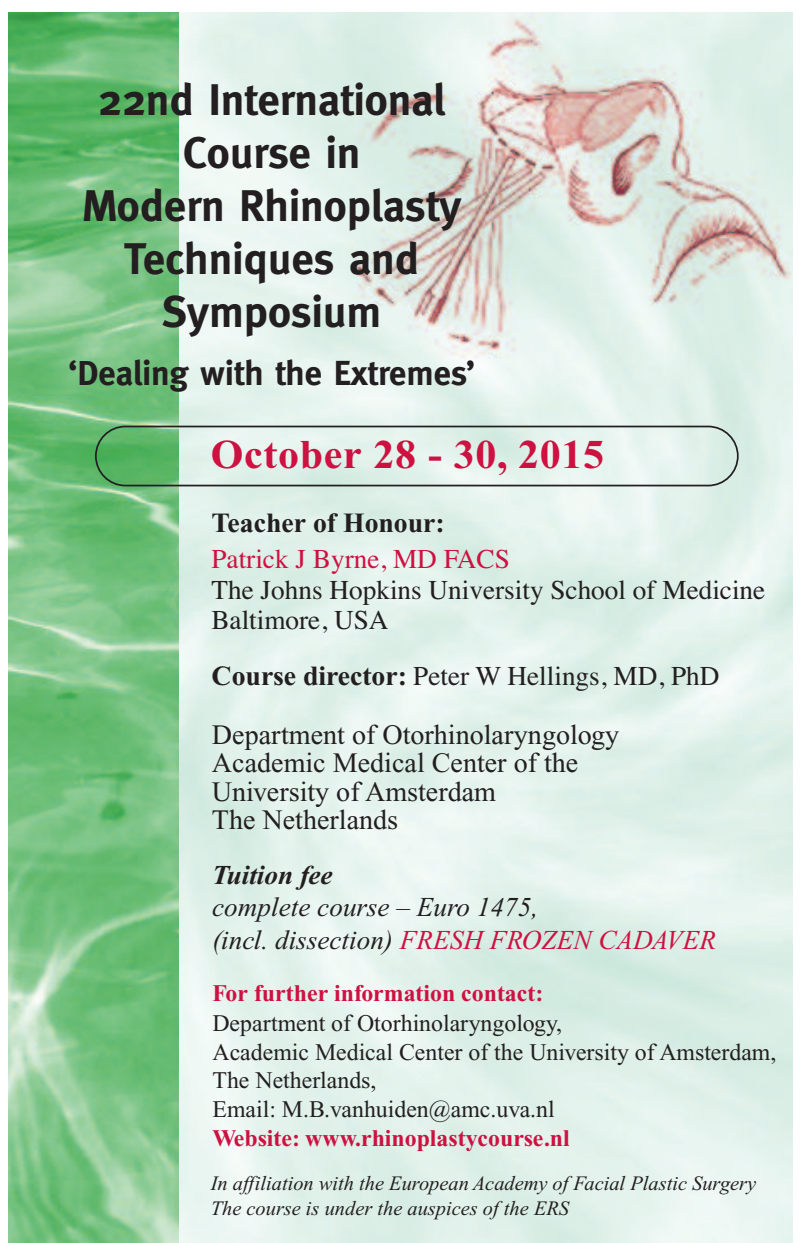

